\title{
BASE CONCEPTUAL DE LA PREPOSICIÓN ENTREY SUS EQUIVALENTES DE LA LENGUA INGLESA BETWEEN, AMONG, Y AMID: UNA PERSPECTIVA EN LINGÜÍSTICA COGNITIVA
}

\section{CONCEPTUAL BASIS OF THE SPANISH PREPOSITION ENTRE AND ITS ENGLISH EQUIVALENT BETWEEN, AMONG AND AMID: A COGNITIVE LINGUISTICS APPROACH}

\author{
Javier Morras Cortés \\ Universidad de Córdoba \\ javiermorrascortes@gmail.com
}

\section{RESUMEN}

El lenguaje espacial es tradicionalmente entendido como un tipo de lenguaje que codifica exclusivamente información geométrica y espacial. Sin embargo, se necesita algo más que tan solo geometría y organizaciones espaciales para una teoría de la semántica espacial que sea psicológicamente real. Para acercarnos a esta, debemos destacar la importancia de consecuencias funcionales (ej., Herskovits 1988 y Vandeloise 1994), que surgen en distintos tipos de organizaciones espaciales. Dichas consecuencias funcionales pueden ser tanto espaciales como no espaciales, lo que pone en evidencia la rígida visión de la geometría y la estructura espacial como las dos únicas estructuras conceptuales codificadas por las preposiciones. Hay también parámetros no espaciales, los cuales, en conjunción con parámetros espaciales, sancionan la semántica de las preposiciones. Esto, a su vez, nos orienta hacia una mejor comprensión de la motivación que hay detrás de los sentidos no espaciales y temporales. La presente investigación intenta indagar en el espectro semántico de las preposiciones propuestas para el español y el inglés; este espectro semántico es aquí entendido como base conceptual. Las bases conceptuales propuestas para entre y sus equivalentes del inglés between, among y amid nos darán un entendimiento de cómo estas preposiciones están organizadas espacialmente y de cómo la estructura esquemática de esta organización es usada para entender y producir expresiones con sentido no espacial y temporal. $\mathrm{El}$ análisis contrastivo también mostrará las diferencias (y similitudes) conceptuales que existen entre los vehículos preposicionales de los dos idiomas. El artículo finaliza con comentarios generales, entre ellos ideas del modelo presentado para su aplicación a la enseñanza del español y el inglés como segunda lengua, así como también para su posible validación experimental en el campo de la psicolingüística.

Palabras clave: base conceptual, parámetros, figura y fondo, trajector/landmark, extensión semántica
Spatial language has been traditionally understood as a type of language that encodes geometric and spatial information exclusively. However, more than pure geometry and spatial organization is needed for a psychologically real account of spatial semantics. To approach this view, we have to take into consideration the importance of functional consequences (e.g., Herskovits 1988, Vandeloise 1994) that emerge from those spatial arrangements. Such functional consequences can be spatial as well as non-spatial, which in turn, amounts to evidence against the vision of geometry and spatial structure as the only two types of information encoded by prepositions. There are non-spatial parameters, which jointly with spatial ones, motivate linguistic-situated events. This interaction leads to a better understanding of the motivation behind non-spatial and temporal conceptions of prepositional vehicles. The present study intends to shed light on the semantic spectrum of the prepositions under examination. This semantic spectrum is referred to as conceptual basis. The conceptual bases proposed for entre and its English equivalents between, among and amid, will provide an account for how these prepositions are spatially rooted and how the spatio-conceptual structure of each preposition is the main motivational factor to conceive non-spatial and temporal usages. This contrastive analysis also shows the conceptual differences (and similarities) that exist among these prepositions. The paper concludes with general remarks, among them the applicability of this model to language teaching, and its possible experimental validation in the field of psycholinguistics

Keywords: conceptual basis, parameters, figure/ground, trajector/landmark, semantic extension 


\section{INT'RODUCCIÓN}

El presente estudio pretende mostrar las diferencias que existen en términos de estructura espacio-conceptual entre la preposición entre y sus equivalentes del inglés between, among y amid. El estudio se centra en hallar los parámetros semánticos que conforman mayoritariamente las bases conceptuales de las preposiciones propuestas, así como, también mostrar cómo estos parámetros se activan de modo diferente dependiendo del contexto lingüístico en el que la preposición se encuentre integrada. Dicho foco investigativo permitirá apreciar una organización espacial más clara de los vehículos preposicionales y mostrará las consecuencias funcionales que surgen de estas configuraciones espaciales. El artículo también apunta a cómo la estructura espacioconceptual de las preposiciones es la fuente motivadora principal de concepciones no espaciales y ayuda a estructurar concepciones temporales.

La investigación es llevada a cabo bajo el foco teórico de algunas teorías y constructos de lingüística cognitiva, filosofía y psicología. Las principales teorías y constructos son los siguientes: gramática cognitiva (Langacker 1987, 1991, 2008), parámetros (Evans 2009, 2010, 2015), esquemas de imágenes (Hampe 2005; Johnson 1987, 2005, 2008; Peña 2012), teoría de la metáfora conceptual (Lakoff \& Jonson 1980, 1999; véase también metáforas primarias en Grady (1997)), teoría de la metonimia (Barcelona 2008, 2015). Estas teorías y constructos serán brevemente definidos en el siguiente apartado.

$\mathrm{El}$ análisis presentado en este artículo está inspirado mayoritariamente en las teorías y constructos mencionados anteriormente. Sin embargo, más constructos e ideas relacionados con lo ya mencionado serán introducidas a lo largo del artículo. Todas estas ideas en conjunto proporcionarán las herramientas necesarias para indagar en las diferencias conceptuales que existen en las preposiciones propuestas para ambos idiomas. A su vez podría servir como sólida referencia lingǘstica para su validación experimental en el campo de la psicolingüística, así como también de herramienta pedagógica para su implementación en el aula.

El artículo está dividido de la siguiente manera. El apartado dos presenta el background teórico necesario para familiarizar al lector con las teorías más relevantes de la lingüística cognitiva usadas en la presente investigación, seguida de la metodología 
tratada. En el tres se presenta el análisis lingüístico en el que se proponen las bases conceptuales para cada preposición y se analizan con el objetivo de proveer una visión en lingüística cognitiva basada en su comportamiento espacial y cómo este sirve como dominio fuente para concepciones no espaciales y temporales (estas últimas con respecto a entre y between ${ }^{1}$ ). Finalmente, el apartado cuatro presenta los comentarios finales acerca de los beneficios del análisis presentado, entre ellos el de su aplicabilidad en el aula, su uso como fuente de estudio y reseña bibliográfica para estudiantes de grado (y avanzados) y su posible validación experimental.

\section{BACKGROUND TEÓRICO Y METODOLOGÍA}

La presente investigación está principalmente basada en la teoría de la gramática cognitiva (GC) (Langacker 1987, 1991 y 2008), al igual que en otras teorías y constructos provenientes de la corriente de la lingǘstica cognitiva. De gran importancia dentro del análisis ofrecido en la siguiente sección, son los constructos de trajector y landmark, propuestos originalmente por Langacker (1987), al igual que los de correspondencias y elaboración (Langacker 1987 y 2008).

Consideremos primero la alineación asimétrica de trajector y landmark que debe ser distinguida de la ya clásica noción de la escuela alemana de psicología de la Gestalt conocida como figura y fondo. El constructo de figura y fondo tiene que ver principalmente con el input perceptual, como cuando le ponemos atención a un pájaro volando por los cielos. El pájaro pasa a ser nuestro foco de atención, el cual es percibido contra fondo, el cielo, montañas o cualquier otro objeto que se encuentre en su background visual. Por otro lado, aunque profundamente ligado a lo perceptual, la organización asimétrica de trajector y landmark es un caso especial de figura y fondo. El trajector de una expresión se puede entender como la figura atencional de un perfil relacional, mientras que otros elementos (también) prominentes son identificados como landmark. La organización asimétrica entre trajector y landmark, tradicionalmente conocida como sujeto/objeto, es

\footnotetext{
${ }^{1}$ Con respecto al comportamiento temporal de among y amid, su uso no es tan frecuente como el de between, por lo que no han sido considerados en el análisis.
} 
considerada en esta investigación, en línea con Langacker (1987), como la instancia lingüística de la habilidad cognitiva de figura y fondo ${ }^{2}$.

Los factores descriptivos de GC, tales como correspondencias y elaboración, son también pieza clave para entender el análisis presentado en el apartado tres. Correspondencias tienen que ver con los links que existen entre unidades simbólicas ${ }^{3}$. Estos son motivados por las subestructuras que las palabras tienen en común. Las subestructuras de las palabras que son semánticamente compatibles pueden ser lexicalmente integradas a través de un ensamble semántico. Elaboración, por otro lado, ocurre cuando un ensamble semántico es puesto en marcha y consiste en destacar, grosso modo, propiedades de predicaciones más autónomas ${ }^{4}$. Como ejemplo, consideremos una expresión como la botella está en la mesa, en donde links de correspondencias son establecidos entre el perfil del nominal la botella y el trajector de en, al igual que entre el landmark de en y el perfil de la mesa. Cuando dichas correspondencias establecen un ensamble semántico, el nominal la botella elabora el trajector de en, mientras que el landmark preposicional es elaborado por la mesa (la preposición en, en este caso, funciona como sitio de elaboración). El compósito estructural resultante designa la botella como principal figura de atención (trajector) y mesa como fondo (landmark).

Otros constructos aplicados al análisis lingüístico del apartado tres son las nociones de parámetros y esquemas de imágenes. En línea con Evans (2009, 2010 y 2015), los parámetros semánticos son un conjunto de información que constituye el polo semántico de una unidad simbólica. Los parámetros en esta investigación son considerados como los elementos que conforman la base conceptual de una determinada palabra, una preposición en este caso. Los parámetros semánticos van de

\footnotetext{
2 Sin embargo, trajector/landmark no subyace sólo la oposición sujeto/objeto. Para ver detalles acerca de trajector/ landmark véase Langacker 1987: 231-242.

${ }^{3}$ Una unidad simbólica en GC es entendida como la relación que existe entre un polo semántico y uno fonético.

${ }^{4}$ En GC, existe lo que se conoce como alineación autónoma/dependiente en la cual sustantivos y nominales como la guitarra, presentan una autonomía conceptual más amplia en comparación a estructuras conceptualmente más dependientes como las preposiciones, verbos, adjetivos, entre otros. Estos funcionan como sitios de elaboración (Langacker 1987) para destacar más a fondo las propiedades de estructuras autónomas que están más involucradas dentro de un perfil relacional.

${ }^{5}$ Para una detallada descripción de los requisitos teóricos en la GC véase Langacker 1987; para detalles en factores descriptivos en GC, véase Langacker 2008:183-215.
} 
la mano de los esquemas de imágenes, los cuales son unidades preconceptuales generalmente relacionadas a nociones espaciales como por ejemplo [CONTACTO], [PRESIÓN], [BALANCE], entre otros ${ }^{6}$.

Los aspectos fenomenológicos, es decir, los elementos de la estructura de la experiencia en todas sus cualidades (Johnson 2005, 2008; véase también Thompson 2010), pasan por un proceso de parametrización, de manera que los elementos fenomenológicos más relevantes dentro de una preposición u otra categoría gramatical constituyen su base conceptual o espectro semántico al cual las palabras ofrecen acceso. La base conceptual, a su vez, permite procesos de elaboración y extensión. Dichos procesos deben ser entendidos como usos lingüísticos situados que lidian con concepciones literales y figurativas respectivamente (pese a que un estricto margen entre lenguaje literal y figurativo es ilusorio dentro de la lingüística cognitiva ${ }^{7}$ ). La parametrización ocurre cuando abstracciones de patrones recurrentes y estructuras que constituyen el contorno básico de nuestra experiencia fenomenológica se arraigan mentalmente y se convencionalizan, al punto de que las complejidades experienciales llegan a ser codificadas a través de ensambles simbólicos (palabras). Estos patrones y estructuras recurrentes, los cuales son conceptuales por naturaleza (ej., no lingüísticos), pasan a conformar el contenido semántico (en forma de parámetros) de la base conceptual de una determinada palabra.

Las últimas dos teorías presentadas brevemente a continuación nos ayudarán a comprender cómo nos movemos de lo espacial a lo "no espacial", de la misma manera que las preposiciones adquieren su semántica situada a través de sus zonas activas (Langacker, 1987, 1991, 2000, 2008 y 2009), que podrían ser guiadas metonímicamente (Barcelona en una comunicación personal; Langacker 2009).

La primera teoría es la de la metáfora conceptual (Lakoff \& Johnson 1980 y 1999), la cual plantea el pensamiento metafórico como pieza clave dentro del sistema conceptual

\footnotetext{
${ }^{6}$ Para ver detalles en cuanto a esquemas de imágenes y su estatus preconceptual véase Hampe 2005; Johnson 1987; Mandler 2004.

${ }^{7}$ En lingüística cognitiva se prefiere asumir un continuo entre lenguaje literal y figurativo. Esta inclinación se argumenta gracias a usos del lenguaje como por ejemplo La tetera está hirviendo, en donde claramente la tetera no es el objeto que está en estado de ebullición, sino que es el agua dentro de esta. En este caso, podríamos hablar de una concepción literal que es extendida metonímicamente.
} 
humano ${ }^{8}$. Las metáforas consisten en una proyección conceptual desde un dominio fuente hacia un dominio meta. Estos dos dominios, han de ser basados en distintas experiencias y son relacionados icónicamente como en la ya famosa metáfora de Lakoff y Johnson love is a journey (el amor es un viaje), en donde se puede ver cómo parte de la estructura fuente [VIAJE] es invariantemente proyectada (Lakoff 1990) al dominio meta $[\mathrm{AMOR}]$. Con la estructura proyectada desde el dominio fuente, el dominio meta puede ser entendido más fácilmente; por ejemplo, los viajeros son conceptualizados en términos de la pareja amorosa, y el recorrido del viaje como el tiempo que lleva la relación, entre las muchas otras proyecciones que puede haber.

También existen metáforas primarias (Grady 1997). Estas están asociadas a correlaciones experimentales que surgen al unísono, como por ejemplo en la metáfora primaria arriba es más (more is up), la cual es evidenciada al llenar un vaso con agua: a medida que llenamos el vaso el agua sube al igual que aumenta la cantidad de esta en el vaso'. Otro ejemplo de metáfora primaria es duración es longitud, en la cual el dominio temporal es conceptualizado en términos espaciales. El concepto primario es [LONGITUD] mientras que el concepto meta es [DURACIÓN] ${ }^{10}$.

En el caso de la metonimia, que es una operación conceptual aparentemente más cotidiana y frecuente que la metáfora (Barcelona 2000; Panther 2006 y Panther \& Thornburg 2009), hay una proyección conceptual de un concepto A a un concepto B. Estos dos conceptos están semánticamente relacionados y establecen una relación indexical en donde A ofrece acceso mental y activa el concepto B. Consideremos otro

\footnotetext{
${ }^{8}$ El sistema conceptual debe ser entendido como el repositorio de conceptos de la mente, el cual está compuesto de modelos cognitivos o marcos semánticos (Fillmore 1982). Este constructo va muy relacionado con el que posiblemente guía las conexiones de los atributos que conforman un marco semántico, el conocimiento enciclopédico (Langacker 1987).

9 Cabe destacar que estas supuestas metáforas primarias podrían ser mejor entendidas como derivaciones metonímicas (Panther 2006; véase también Barcelona 2000) puesto que manifiestan más indexicalidad que iconicidad.

${ }^{10}$ Sin embargo, cabe mencionar el estatus especial del dominio temporal frente a otros dominios no espaciales, y es que el dominio temporal presenta características fenomenológicamente reales y bases neurobiológicas (Evans 2013; Galton 2011). De hecho, espacio y tiempo pueden ser detalladamente analizados bajo los parámetros de magnitud, dimensionalidad y direccionalidad. La información espacioconceptual es un complemento para la estructura temporal. Espacio y tiempo poseen sus propias estructuras puesto que son cualitativamente distintos (para manifestaciones lingüísticas de espacio y tiempo véase Langacker 2012a).
} 
clásico ejemplo tomado de Lakoff y Johnson (1980) para ver cómo funciona la metonimia. En la expresión the ham sandwich asked for his check (el emparedado de jamón pidió la cuenta), que puede ser dicha en un restaurante por un mesero a otro, podemos apreciar cómo el emparedado de jamón (concepto A) brinda acceso mental (y activación) a la persona que ordenó el sándwich (concepto B). Estos dos conceptos, a su vez, están semánticamente e indexicalmente relacionados bajo un marco o rol semántico de \{RESTAURANTE\} (Schank \& Abelson 1977).

Existen también metonimias más esquemáticas (Barcelona 2008, 2011 y 2015) que van relacionadas con la activación de los parámetros que están más involucrados en un determinado evento lingüístico. Por ejemplo, en una oración como las nubes sobre la cuidad, se observa que la preposición sobre se comporta de una manera atemporal simple (Langacker 1987 y 2008), en el sentido de que denota una relación estática entre las nubes (trajector) y la cuidad (landmark). En la figura 1 mostrada abajo, se conceptualizan las nubes como el punto B mientras que los límites de la cuidad estarían conformados por los puntos A y C.

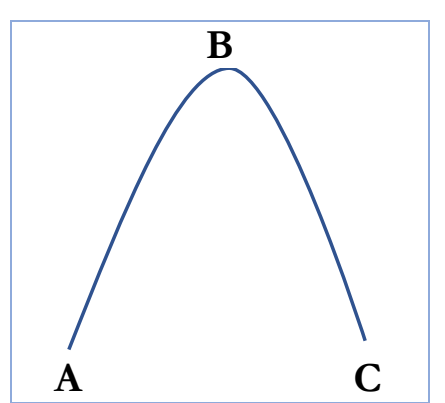

FIgURA 1. Estructura esquemática situada de sobre y sus posibles activaciones

Consideremos ahora una oración como el gato saltó sobre la muralla para llegar al río, en la cual la preposición sobre ya no actúa como unidad relacional atemporal de carácter simple, sino de carácter complejo, puesto que hay escaneo secuencial en vez de sumario. En este caso, el gato (trajector) empieza en un punto A y luego salta la muralla (punto B) para finalmente llegar al río (punto C).

Estos dos ejemplos apuntan a que la preposición sobre designa una relación espacial cuya zona de activación es activada metonímicamente. En línea con Langacker (1987), las palabras son entendidas como puntos de acceso a la estructura conceptual, la cual es 
semánticamente codificada a través de la base conceptual de una determinada palabra. Una vez dentro de la base conceptual, patrones metonímicos dirigen la activación de las zonas que están más involucradas en un evento lingüístico situado, como se ha ejemplificado en el introductorio caso de sobre ${ }^{11}$.

\subsection{Metodología}

La metodología que se llevará a cabo para analizar las preposiciones entre, between, among y amid será la de presentar su proto-escena (siguiendo a Tyler \& Evans 2003) ${ }^{12}$ e indicar los parámetros que podrían conforman su núcleo semántico o base conceptual. Los parámetros propuestos no son, bajo ningún tipo de circunstancia, estrictos ni absolutos para cada base conceptual, puesto que aquella visión sería menospreciar el potencial semántico (Allwood 2003) del cual las palabras proveen acceso (Taylor 2006). El presente análisis solamente pretende mostrar, con una idea de base conceptual, el potencial comunicativo que tienen las preposiciones (y las palabras, en general) en los procesos de elaboración y extensión. Un potencial semántico que podría activar una proliferación de sentidos distintos, en especial, en contextos nuevos. Dicho potencial, entendido aquí como base conceptual, será también respaldado con evidencia lingüística real, ya que la mayoría de los ejemplos en el apartado tres han sido extraídos desde dos bases de corpus a través de una búsqueda manual. Para la preposición española entre, se presentarán ejemplos obtenidos de la base Spanish Web 2011 (esTenTen11, Eu + $A m)^{13}$, mientras que para las preposiciones inglesas se ha hecho uso del British National Corpus (BNC). Los diccionarios Cambridge English Dictionary (2008) y Diccionario de la Lengua Española (DEL 2014) también son utilizados.

\footnotetext{
${ }^{11}$ Para ver detalles acerca de influencias metonímicas en la gramática véase Langacker (2009). Para una propuesta acerca de la posibilidad de motivaciones metonímicas en metáfora conceptual véase Barcelona (2000).

12 Tyler \& Evans (2003) proponen el constructo de proto-scene para referirse al espacio físico en el cual los seres humanos adquirimos abstracciones de patrones estructurales recurrentes como son las relaciones espaciales que son codificadas por preposiciones y partículas (entre otras categorías léxicogramaticales).

${ }^{13}$ Disponible en línea en: https: / / auth.sketchengine.eu/?next=https $\% 3 \mathrm{~A} \% 2 \mathrm{~F} \% 2 \mathrm{Fapp}$. sketchengine.eu $\% 2 \mathrm{~F} \% 3 \mathrm{~F}$ corpname $\% 3 \mathrm{D}$ preloaded $\% 2$ Festenten 11 fl5\%26struct attr stats $\% 3$ D $1 \% 26$ subcorpora $\% 3$ D1 [Consultado 12-122018].
} 
El análisis lingüístico proveerá ideas para un mejor entendimiento del fenómeno conceptual conocido como polisemia, ya que muestra cómo los parámetros son activados dependiendo del contexto lingüístico. En otras palabras, se observará las zonas activas de las bases conceptuales bajo contextos literales, figurativos y temporales ${ }^{14}$. También se verá similitudes y diferencias conceptuales entre los vehículos preposicionales analizados. El análisis comenzará con las preposiciones inglesas puesto que el campo semántico de entre es, como veremos a lo largo del análisis, más amplio debido a que cubre prácticamente todo el campo semántico que between, among y amid hacen separadamente.

\section{ANÁLISIS}

\subsection{BETWEEN}

La preposición del inglés between se caracteriza por designar una relación espacial entre un trajector que está ubicado entre dos o más entidades que conforman su landmark. A continuación se presenta algunas proto-escenas que podrían dar lugar a la parametrización de los elementos semánticos que forman su base conceptual:

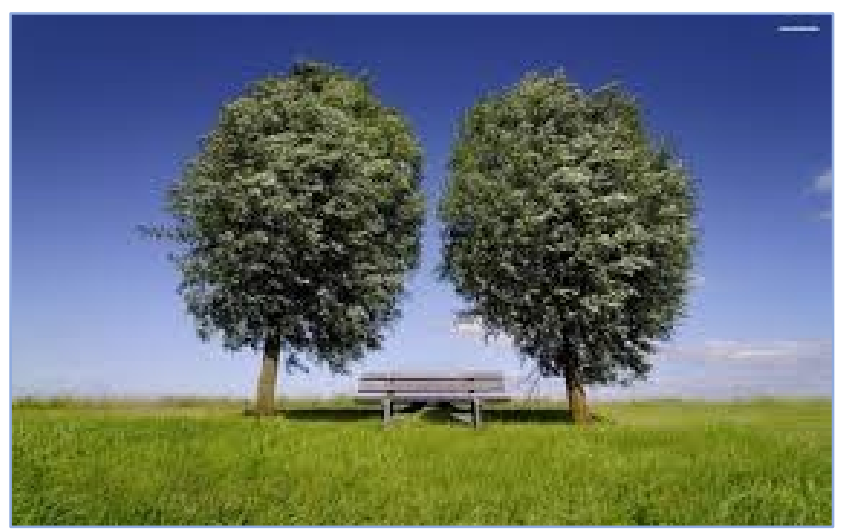

IMAGEN 1. Proto-escena de between. "A bench between two trees" (un banco entre dos árboles)

En la imagen 1 de arriba, podemos localizar el banco con respecto a los dos árboles. Nótese que, si los dos árboles estuviesen a un mismo lado, la expresión un banco entre

\footnotetext{
${ }^{14} \mathrm{El}$ presente autor se abstiene de referirse al dominio temporal como "figurativo" debido a su estatus especial en la cognición humana. El dominio temporal aparentemente es más básico que el espacial. Después de todo, cada evento y pensamiento, ocurren en tiempo de proceso y tiempo concebido (Langacker 1987).
} 
dos árboles no podría ser usada, puesto que no cumpliría con el factor fenomenológico de un foco de atención el cual está posicionado con respecto a un objeto a cada lado (ej. izquierda y derecha). El banco, en la imagen 1, está separado físicamente de los árboles, pero podría estar pegado a ellos y, aun así, diríamos un banco entre dos árboles, ya que conceptualmente podemos distinguir objetos y interactuamos con ellos en el espacio.

En el Cambridge English Dictionary (2008) la preposición between denota una persona o cosa que está ubicada en el espacio que separa dos lugares, personas u objetos. Consideremos un ejemplo lingüístico extraído de la British National Corpus (BNC) para ejemplificar este sentido prototípico y posteriormente proponer una base conceptual para between:

(1) There is a thick mist between the hills and us. I Can't see through it, but through it we shall have to go

(Hay una gruesa niebla entre los cerros y nosotros. No puedo ver a través de ella, pero aun así por ahí debemos ir)

[LOCACIÓN]

En (1) podemos observar que en el compósito estructural ${ }^{15}$, a thick mist between the bills and us (una gruesa nieblas entre los cerros y nosotros), el perfil del nominal de a thick mist corresponde con el trajector de between, mientras que el perfil de la estructura de coordinación the hills and us (los cerros y nosotros) establece correspondencias y elabora el landmark preposicional. En (1) el landmark está constituido por yuxtaposición mental (Langacker 2008) de dos elementos, en este caso el "grupo de cerros" y el de "personas", mientras que el trajector es la "niebla" que está posicionada entre el grupo de personas y los cerros.

Ahora consideremos una propuesta de base conceptual sobre la manera de entender los atributos semánticos que más involucrados están en el ejemplo (1):

\footnotetext{
${ }^{15}$ Compósito estructural (siguiendo a Langacker 1987, 2008) se refiere a la integración lexical parcial que es posteriormente interpretada y entendida como concepción del compósito (traducida de composite conception) (sin embargo, véase Evans 2009: c.13 para ver detalles acerca de la interpretación).
} 


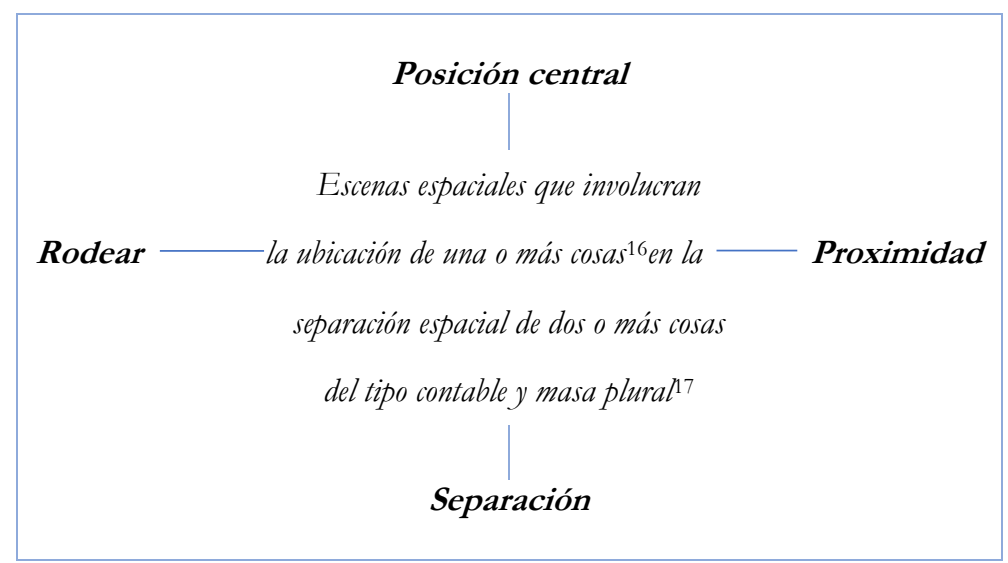

FIGURA 2. Base conceptual propuesta para between

La figura 2 de arriba, muestra una posible base conceptual de between, la cual es originada por proto-escenas (indicadas en el centro con itálicas) que dan origen a abstracciones de estructuras y patrones experienciales concurrentes que son parametrizados y pasan a conformar el núcleo semántico de between.

La preposición inglesa between proporciona acceso a su base conceptual de la que algunas zonas conceptuales o parámetros se activan dependiendo del contexto lingüístico -el uso situado de la preposición. En el caso del ejemplo (1) podemos observar que la zona activa de la base conceptual de between está en los parámetros de posición central, proximidad y separación. La niebla tiene una posición central dentro de la delimitación física (y mental) creada por los cerros y el grupo de personas, estos tres elementos son conceptualizados separadamente. También existe proximidad entre trajector y landmark, en este caso, el grupo de personas puede observar la densa neblina que existe entre ellos y los cerros.

Consideremos brevemente otro ejemplo de between extraído de la $B N C$ :

(2) Further away, they could see a bigh bridge between the bills. But the station was too far away to see (Mas allá, ellos pudieron ver un alto puente entre los cerros. Pero la estación aún estaba muy lejos para ser vista)

[Locación]

\footnotetext{
${ }^{16}$ Cosas debe ser entendido bajo su estructura esquemática de \{COSAS\} que está presente en el perfil de personas y objetos (concretos y abstractos).

${ }^{17}$ La clasificación de sustantivos usada en esta investigación está basada en Langacker (2008: 128-147).
} 
Lo primero que se observa en (2) es que también sanciona el sentido de [locación] al igual que en (1); sin embargo, la conceptualización resultante conlleva a una simulación mental distinta ${ }^{18}$. El compósito estructural a high bridge between the bills (un alto puente entre los cerros) tiene como trajector high bridge y como landmark the hills, los cuales elaboran el trajector y el landmark de between respectivamente. Between sirve como sitio de elaboración, convirtiéndose en la unidad relacional entre trajector y landmark a un mayor nivel estructural.

La activación de parámetros que recibe between es la de rodear y proximidad; sin embargo, no se descarta la posibilidad de activaciones secundarias (idea inspirada en spread activation (Langacker 2012b) y secondary activation (Evans 2009)) de los otros parámetros. Activación primaria concierne a los parámetros que se ven más involucrados en un uso lingüístico situado. La activación primaria de rodeo se refiere a la capacidad de algunos elementos que elaboran el landmark de between (y de entre) de poder rodear al determinado trajector, como es el caso del nominal the hills ${ }^{19}$. El puente en (2) podría estar rodeado de cerros, considerando que está entre ellos. También existe proximidad entre los cerros y el puente, al punto de que dicha proximidad es también contacto en el comienzo y término de este.

\subsubsection{Extensión semántica de between}

Consideremos ahora casos de figuración en los que between se ve involucrado. Los ejemplos son extraídos desde la BNC:

(3) In fact, between you and me, I think she's relieved [secreto] (De hecho, entre tú y yo, yo creo que ella esta aliviada)

(4) We can make a choice between struggling on or surrendering forever to the forces of death (Podemos elegir entre luchar o rendirse para siempre ante las fuerzas de la muerte)

[decisión]

\footnotetext{
18 Para revisar literatura acerca de simulaciones mentales, véase Barsalou (1999); Bergen (2012); Evans (2009); Gallese \& Lakoff (2005); Zwaan (2004), y referencias en ellos.

19 Cabe agregar que el landmark preposicional constituido por the bills carece de estructura de coordinación del tipo -y como en (1). Esto podría estar ligado a la activación del parámetro de separación cuando encontramos construcciones en las que el landmark de between es elaborado por una estructura de coordinación, y activación en rodeo cuando el landmark preposicional no involucra yuxtaposición mental (para una breve descripción de estructuras de coordinación del tipo - $y,-0, y$-pero, véase Langacker 2008:406-412).
} 
El ejemplo (3) muestra cómo between puede establecer una relación entre dos personas, y algo imperceptible, como es un secreto. La concepción resultante de dicho compósito estructural es la de [secreto]. En (3), el trajector de between es elaborado por you and me mientras que su landmark también, luego, a un mayor nivel estructural, es integrado con I think she's relieved, el cual pasa a ser el determinador del perfil y elabora el trayector, dejando como landmark a la estructura de coordinación you and me (tú y yo). Nótese cómo un secreto que es sabido por dos personas es conceptualizado como algo que puede estar "entre ellos". Si volvemos a la figura 2, podemos ver que hay activación del parámetro de posición central, el cual es extendido (y posiblemente activado metonímicamente). También puede haber activación de separación, como manera de apoyar la proyección metafórica con su estructura esquemática y poder concebir una cosa abstracta que es compartida entre dos personas.

En el ejemplo (4), podemos ver que el landmark preposicional de between está compuesto por una estructura de coordinación del tipo -0, la cual es struggling on or surrendering forever (luchar o rendirse por siempre), mientras que el trajector es elaborado por make a choice (decidir). La activación primaria de zonas conceptuales de between, ocurre principalmente en el parámetro de separación, puesto que esto permite la conceptualización de dos opciones a elegir. También puede haber activación secundaria de posición central, el cual podría ayudar a metafóricamente razonar acerca de una "perspectiva" desde donde podemos contemplar las opciones que se nos presentan. En el supuesto caso de decir a Juan se le presentaron múltiples oportunidades para trabajar este mes, podríamos conceptualizar al sujeto metafóricamente como rodeado de oportunidades para su elección. En (4) hay solo dos, sin embargo, la locación central de un objeto atencional en la proto-escena de between, contribuye en la estructura esquemática que es proyectada del dominio espacial (fuente) al dominio no espacial (meta).

\subsubsection{Comportamiento temporal de between}

En esta subsección procederemos a analizar brevemente el comportamiento temporal de between. Para ello, consideremos el siguiente ejemplo extraído de la BNC: 
(5) A study published in the British Medical Journal traces the excess cesium to locally produced lamb and milk from cows which have eaten coastal grasses contaminated with nuclear waste. The measurements were taken between 1979 and 1986.

(Las medidas fueron tomadas entre 1979 y 1980)

[demarcación temporal]

En (5), se ha marcado en itálicas y traducido al español la parte de mayor interés para el presente análisis, que se refiere a "las muestras de unos elementos químicos que fueron tomados entre los años indicados". La preposición between funciona como elemento relacional temporal entre el trajector y el landmark, los que corresponden con los perfiles de measurements y 1979 and 1986, respectivamente. Nótese cómo esta concepción temporal comparte estructura esquemática con construcciones que invocan concepciones espaciales, como en (1). Los ejemplos (1) y (5) no solamente son similares a nivel conceptual, sino que también a nivel gramatical, puesto que, en ambos ejemplos, el landmark preposicional de between es elaborado por una estructura de coordinación del tipo -and (y).

La proyección metafórica de estructura esquemática (invariante) del dominio espacial al temporal, debe ser visto como un complemento en el que la estructura espacio-conceptual ayuda a especificar en mayor grado la estructura temporal. Recordemos que el tiempo tiene su propia estructura (Evans 2004, 2013; véase también Galton 2011). Por ejemplo, necesitamos el concepto temporal de [año], al igual que hacer uso del marco temporal absoluto, el cual se basa en nuestra capacidad de pensar acerca de periodicidades (Evans 2013: c.6). Mas aún, para simular mentalmente que dichos hechos en (5) tomaron lugar entre esos años, necesitamos conceptualizar el tiempo linealmente dentro de un sistema de reconocimiento de eventos.

\subsection{AMONG}

Among se comporta diferente a between, principalmente en que necesita al menos tres elementos (los cuales no son explícitamente mencionados ${ }^{20}$ ) o algunas substancias como hiedra o arena, para que desarrollen su landmark como por ejemplo en among the weed (entre la hiedra). Los elementos elaboradores del landmark de among están

\footnotetext{
${ }^{20} \mathrm{Al}$ comparar expresiones como: there are good relationships between Uruguay, Chile, and Brazil, a there are good relationships among south American countries. El landmark de between ofrece una mayor especificación que el de among.
} 
generalmente relacionados con sustantivos del tipo masa plural (ej. diamantes) y masa no plural (ej. oro), mientras que los de between con contable (ej. diamante) y masa plural.

En el Cambridge English Dictionary (2008), among denota una cosa o cosas que están en medio o rodeadas de otras cosas. Consideremos un ejemplo de esta denotación extraído de la $B N C$ :

(6) the path and the valley are actually a trap created by a flock of Harpies who nest in a small cave hidden among the rocks.

(el camino y el valle son una trampa creadas por hadas que viven en nidos en pequeñas cuevas escondidas entre las rocas)

[Locación]/[Oclusión]

Podemos observar en (6) que el compósito hidden among the rocks (escondidas entre las rocas) destaca la locación de los nidos de hadas, como también la oclusión de estos puestos que se encuentran escondidos en un área rocosa. Si vemos la figura 3 de abajo, podemos observar la base conceptual propuesta para among. Nótese la activación primaria de los parámetros rodear, oclusión y homogeneidad (y posible activación secundaria de inclusión puesto que las cuevas de hadas en (6) forman parte de una mayor organización espacial que es su hábitat rocoso). Rodear apunta a las condiciones en donde están los nidos, que son rodeados por rocas en una cueva. Oclusión tiene que ver con la consecuencia funcional de estar rodeado de algún tipo de material no transparente. Finalmente, homogeneidad apunta a la naturaleza conceptual del elemento que elabora el landmark preposicional de among. Conceptualizar un espacio rocoso implica homogeneidad puesto que hablamos de muchas instancias de un mismo tipo.

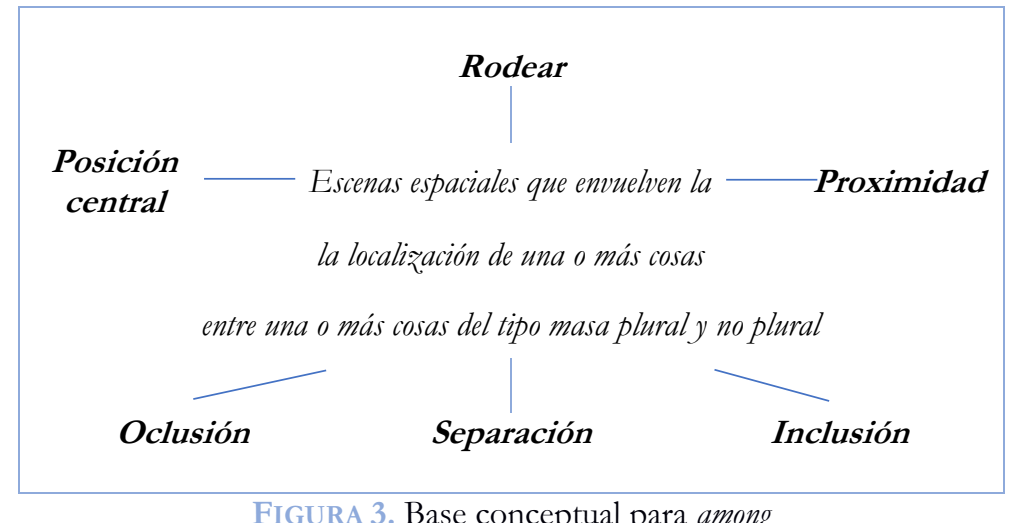

FIgURA 3. Base conceptual para among

En la figura 3 de arriba, la proto-escena de among (mostrada en el centro) denota una organización espacial como la mostrada en la imagen 2 abajo, en donde la manzana verde 
(figura) es localizada con respecto de las rojas (fondo). Nótese las siguientes características exhibidas por el fondo o manzanas rojas: (1) ellas rodean a la manzana verde; (2) la ocluyen parcialmente; y (3) hay homogeneidad entre las manzanas rojas. Por otro lado, la figura de atención o trajector, si nos referimos a una expresión como una manzana verde entre muchas rojas (a green apple among many red ones), exhibe proximidad (al punto que hay contacto) frente a su landmark y es también incluido como parte de un grupo mayor de manzanas. Por último, el hecho de que la manzana verde esté rodeada los 360 grados por manzanas rojas hace que esta adquiera una position central.

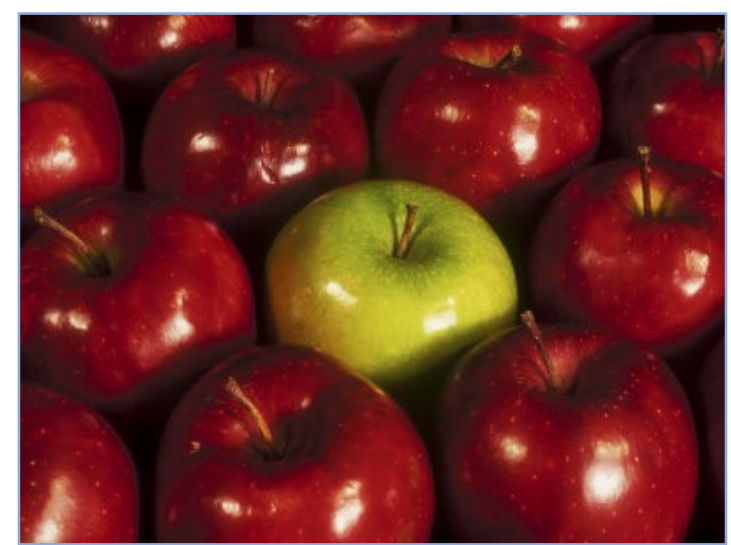

IMAGEN 2. Posible proto-escena de among

\subsubsection{Extensión semántica de among}

Ahora procederemos a ver un par de usos figurativos de la preposición inglesa among. Ambos ejemplos han sido extraídos de la $B N C$ :

(7) The models also describe the exact form of the deterioration in decision making which occurs when people have to choose among several alternatives instead of just two

(Las modelos también describen la forma exacta de deterioro en el momento de tomar decisiones, las cuales ocurrieron cuando la gente tuvo que elegir entre muchas alternativas, en vez de solo dos)

[GRUPO/SELECCIÓN]

(8) Among her leisure occupations is birdwatching

[MIEMBRO DE UN GRUPO]

(Entre sus ocupaciones de ocio, está la de mirar aves.)

En (7), la preposición inglesa among en el compósito semántico integrado por choose among several options (elegir entre muchas opciones), funciona como elemento conceptual relacional entre el evento de hacer una elección y las opciones que hay 
disponibles para ello. La base conceptual de among propuesta en la figura 3 de arriba, podría ayudarnos a entender la motivación que hay detrás de choose among several options y su semántica de [GRUPO/SELECCIÓN]. Los conceptos [SELECCIÓN] y [GRUPO], designados por choose y several options respectivamente, elaboran el trajector y el landmark de among en (7). Los parámetros que podrían estar involucrados en dicha relación son rodear y homogeneidad. Como se ha indicado anteriormente, rodear ayuda a conceptualizar escenas no espaciales en las cuales podemos elegir cosas que no necesariamente están a simple vista. Una hipotética simulación de cómo una expresión como elegir entre muchas opciones podría ser conceptualizada, es mostrada en la figura 3 abajo.

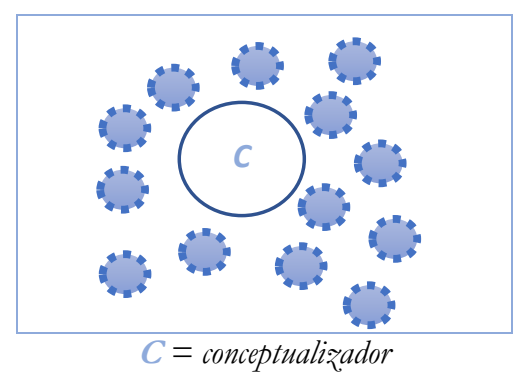

Figura 4. Posible simulación de among en (7)

El parámetro de homogeneidad también está presente considerando que several options (muchas opciones) designa un grupo constituido por instancias de un mismo tipo.

Con respecto a (8), la contribución semántica de among es similar a (7) ya que ambos landmarks evocan una agrupación conceptual. Sin embargo, el parámetro que más directamente tiene que ver con la semántica situada de among en (8) es el de inclusión, puesto que la actividad de ver aves es una actividad de ocio dentro de las tantas que una persona puede tener. La categoría en este caso de \{ACTIVIDADES DE OCIO\} destaca una actividad mientras conserva el resto de las actividades sin designar. El compósito estructural among her leisure occupations is birdwatching establece primero (en el caso de que las simulaciones mentales fuesen lineales) el espacio mental basado en la categoría \{ACTIVIDADES DE OCIO\} seguida por una designación de un miembro de esa categoría.

La figura 5 de abajo, muestra patrones esquemáticos de construcción de significado. El círculo exterior representa la categoría de $\{$ ACTIVIDADES DE OCIO $\}$, mientras que el 
círculo interno representa el miembro designado que cumple el rol de determinador del perfil ${ }^{21}$ en (8).

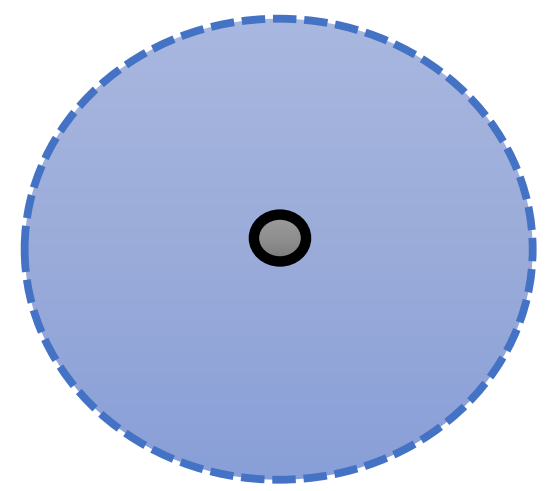

Figura 5. "Entre sus actividades de ocio, se encuentra la de observar aves"

En la figura 5, la línea segmentada del círculo exterior simboliza su contenido de "soporte conceptual"; esto quiere decir que es el background, o fondo conceptual, frente al cual se puede entender el foco atencional. En ese espacio mental se encuentran todas las actividades de ocio que no fueron mencionadas en (8). En cambio, en el espacio mental interior, al cual le podríamos atribuir una relación recursiva con respecto al espacio mental establecido previamente, se le atribuye un rol más atencional, puesto que es el miembro que es seleccionado para servir de ejemplo frente a la categoría a la cual pertenece, y que también cumple un rol prominente en la concepción del compósito estructural en (8). Este rol más atencional de birdwatching (observar aves) es representado por la línea gruesa. Su estatus de figura hace que el perfil de birdwatching establezca lazos de correspondencias con el trajector de among para posteriormente elaborarlo.

\subsection{Amid}

Ahora procedemos a analizar la preposición inglesa amid, la cual está semánticamente relacionada con between y among (especialmente con among), pero que, sin embargo, ofrece características conceptuales propias. Una de ellas es la de unificación

${ }^{21}$ Para ver detalles en determinador de perfil, véase Langacker (2008:192-198) 
conceptual $^{22}$, la cual se ve reflejada en los elementos que elaboran el landmark preposicional de amid. Esta preposición es también mayoritariamente usada con sustantivos abstractos, como se evidencia en una simple búsqueda de concordancia en corpus. A amid se le puede atribuir no solamente un uso más ligado al lenguaje escrito y formal, sino que también presenta una tendencia en la cual su landmark preposicional es elaborado por sustantivos abstractos.

De acuerdo con el Cambridge English Dictionary (2008), amid denota una cosa o cosas que se encuentran posicionadas en medio o rodeadas de uno o más elementos. Dicha definición en este diccionario también es compartida con among. El presente autor concuerda con esta decisión y agrega que las proto-escenas de among podrían ser compartidas en parte con las de amid, debido a que los parámetros semánticos de rodear y homogeneidad son compartidos entre estas preposiciones inglesas ${ }^{23}$. Evidencia lingüística de dicho patrón conceptual es captada por el siguiente ejemplo extraído de la $B N C$ :

(9) A koliba turned out to be a large wooden chalet-type restaurant which in this case was set amid tall pine trees.

(Un koliba resultó ser un restaurante grande de madera tipo chalé, el cual, en este caso, fue construido entre/en medio (de) altos pinos.)

[EN MEDIO DE]

En (9) podemos observar que la ubicación del koliba, el cual elabora el trajector de amid, es establecida gracias a los elementos que elaboran el landmark preposicional, en este caso, tall pine trees (pinos altos). Como ya se ha mencionado anteriormente, el parámetro de rodear recibe activación primaria puesto que el restaurante se encuentra en medio de o entre muchos árboles que lo rodean. Los árboles a su vez son conceptualizados unitariamente, como un conjunto que puede rodear al elemento ubicado en esta área poblada por altos pinos.

La figura 6 de abajo corresponde a una propuesta de base conceptual para amid:

${ }^{22}$ Para ver detalles acerca del concepto de unificación (que proviene del término inglés unitization) véase Langacker (2008: 342-346).

${ }^{23}$ Las palabras que compartan los mismos parámetros podrían presentar superposición semántica, la cual no debería ser comprendida rígidamente, sino que situadamente. Los parámetros semánticos de cada palabra tienden a acomodarse a su semántica contextualizada. 


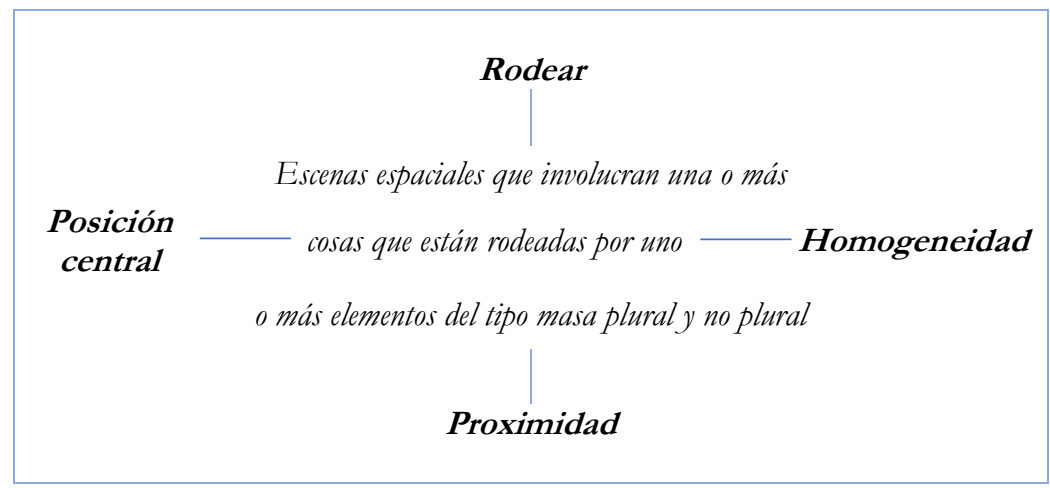

FIGURA 6. Base conceptual propuesta para amid

Como podemos observar en la figura 6 , las escenas espaciales que involucran un objeto rodeado son las que podrían dar pie a la parametrización de los cuatro elementos capaces de conformar el núcleo semántico de amid. Más aún, estos objetos (ej. los que elaboran el trajector de amid) son rodeados por cosas del tipo masa. Esta última característica, presentada por el landmark preposicional de amid, es una similitud semántica que tiene con el de among $^{24}$.

La posición central surge como consecuencia de una organización espacial en la que la figura de atención es rodeada por otros elementos. La homogeneidad, al igual que en among, es una característica que el landmark preposicional de amid presenta frente a los elementos que lo desarrollan. El parámetro de proximidad está reflejado en que el trajector es localizado con respecto a su landmark. La proximidad en este caso apunta a la localización del trajector dentro del área establecida por su landmark como en amid the desert (en medio del desierto). Finalmente, el ultimo parámetro que considero esencial en la base conceptual de amid es rodear, el cual es un elemento semántico que surge como consecuencia de una figura atencional rodeada por un fondo conceptual del tipo masa.

Estos cuatro parámetros podrían ayudar a comprender de mejor manera las instancias de elaboración y extensión de amid, como por ejemplo en (9), donde los

\footnotetext{
${ }^{24}$ El landmark preposicional de among también presenta unificación, como en among the crowd (entre la multitud), en donde los individuos no son lo que el sustantivo multitud designa, sino el conjunto de ellos conceptualizado como una masa de gente.
} 
parámetros con mayor activación son rodear y homogeneidad. Los parámetros de posición central y proximidad podrían recibir activación secundaria.

\subsubsection{Extensión semántica de amid}

A continuación, presento dos casos de figuración de amid. Ambos ejemplos han sido extraídos del BNC:

(10) Amid the gloom and doom, the American film industry rather charitably nominated Hello, Dolly! for seven Oscars, including one for Best Film

(Entre la penumbra y la fatalidad, la industria americana de películas, muy caritativamente nominó Hello, Dolly! a siete óscars, incluyendo uno a la mejor película) [TÉRMINO MEDIO]

(11) Amid mounting criticism of the French government's failure to speak out against the killings, French Foreign Ministry spokesman Daniel Bernard on Jan. 13 condemned the attacks on the opposition and said that France would only continue to support the government

(Entre/ en medio de crecientes criticas)

[EN MEDIO DE/ENTRE]

En el ejemplo (10) podemos notar como amid contribuye con la semántica de [TÉRMINO MEDIO], puesto que establece una relación con los elementos conceptualmente más autónomos, me refiero a \{PENUMBRA\} (gloom) y \{FATALIDAD\} (doom), los cuales conjuntamente, elaboran el landmark preposicional de amid mientras que \{NOMINACIÓN AL OSCAR\} elabora su trajector. La concepción resultante es la localización y relación del trajector respecto a estas dos sensaciones que conforman estados mentales.

Una cosa que hay que destacar frente a este uso de amid en (10), es que al parecer no puede ser reemplazado ni por between ni por among. El carácter conceptual de amid con los elementos que elaboran su landmark, especialmente elementos del tipo masa no plural como penumbra y fatalidad, define situaciones de uso como esta, en la cual a un evento se le destacan sus cualidades experienciales o estética fenomenológica (Johnson 2008).

Con respecto a las zonas activas en la base conceptual de amid, se podría considerar al parámetro de posición central como el más involucrado en la concepción del compósito estructural amid the gloom and doom. El parámetro de posición central es elaborado y extendido de manera que es interpretado en el domino no espacial gracias a la 
estructura esquemática provista. El de homogeneidad podría recibir activación secundaria, puesto que los elementos que elaboran el landmark preposicional son compuestos por una misma "substancia abstracta". Sin embargo, una vez que el trajector es especificado por la nominación al Óscar, estas substancias son mezcladas debido a que son características presentadas en el trajector. Dicha mezcla conceptual a su vez debe ser entendida en el sentido de Fauconnier y Turner (2002), en donde el trajector adquiere atributos de dos espacios mentales distintos, lo cuales son proyectados a un espacio mental mezclado.

En el ejemplo (11), del cual solamente fue traducida la parte de interés debido a razones de espacio, el compósito amid mounting criticism (entre/ en medio de crecientes críticas), apunta a una situación del gobierno francés en la cual el representante del Ministerio de Exteriores tuvo que hacer algunas declaraciones (dicho escenario elabora el trajector de amid en (11)). Esta situación, a su vez, va relacionada con el landmark preposicional de amid, el cual es mounting criticism y apunta a la estética fenomenológica del trajector (ej., sensaciones y emociones). El evento en (11) se sitúa en un contexto de críticas crecientes. Nótese que a diferencia de (10), el ejemplo (11) no presenta una mezcla de cualidades para el trajector, sino un solo concepto abstracto (ej., críticas). Los parámetros que podrían verse involucrados más directamente son posición central, homogeneidad y posiblemente rodear (o puede que este último reciba activación secundaria), ya que ayudan a posicionar metafóricamente el escenario de declaraciones en (11) como algo que está en medio de la masa de críticas crecientes que hay frente a una determinada maniobra del gobierno francés.

\subsection{DifERENCIAS Y SIMILITUDES CONCEPTUALES ENTRE BETWEEN, AMONG Y AMID}

Antes de presentar la base conceptual de la preposición entre, se indicarán brevemente algunas diferencias conceptuales entre las preposiciones del inglés analizadas. Este breve resumen pretende facilitar una comparación posterior con la preposición entre, principalmente para ver cómo entre cubre prácticamente todo el campo semántico que between, among y amid cubren separadamente. 
Lo primero que se nota en estas tres preposiciones inglesas es su similitud semántica al ubicar un trajector con respecto a uno o más elementos. Sin embargo, los elementos que elaboran el landmark de cada preposición son conceptualmente distintos. En el landmark preposicional de between, los elementos que lo elaboran suelen ser sustantivos contables y masa del tipo plural, mientras que los landmarks de among y amid suelen ser elaborados por sustantivos del tipo masa plural y masa no plural.

Otro rasgo que quiero destacar frente a estas preposiciones inglesas es su carácter conceptual, el cual hace posible una diferenciación semántica (como se ha intentado en el análisis hasta ahora). Dicho carácter está determinado por los parámetros semánticos de sus bases conceptuales. En el caso de between, su carácter conceptual está mayoritariamente relacionado con el parámetro de separación, propiedad conceptual clave para entender organizaciones espaciales (prototípicas) designadas por between. Este carácter conceptual está relacionado con la capacidad del landmark de between de ser elaborado por sustantivos contables, como en the remote is between you and $m e^{25}$ (el control remoto está entre tú y yo). En el caso de among, su carácter conceptual podría provenir del parámetro de inclusión, como en she's among her friends (ella está entre sus amigos), en el cual ni amid ni between podrían reemplazar esa función semántica. Por último, el factor conceptual que le da carácter e identificación a amid, es el de posición central puesto que esta preposición enfatiza el concepto de [EN MEDIO DE], como por ejemplo en amid the desert (en medio del desierto) o en amid the gloom and doom (entre la penumbra y la fatalidad), mostrado en (10) anteriormente. En ese ejemplo particular, de hecho, no se podría llegar a la misma concepción ([TÉRMINO MEDIO]) usando una preposición alternativa como among o between. Esto a su vez, va relacionado con la capacidad del landmark preposicional de amid de ser elaborado (principalmente) por sustantivos del tipo masa no plural (y masa plural).

El último punto que se destaca de estas tres preposiciones, y que está indicado en la tabla 1 debajo, concierne el nivel lingüístico en el que between, among, y amid son integrados. Aquí la composición de los landmarks preposicionales están basados en

\footnotetext{
${ }^{25}$ Nótese que la misma expresión usando among o amid sonaría semánticamente anómala como en *the romote is among/amid you and me.
} 
estructuras de coordinación del tipo $-y,-0$, o la ausencia de estas estructuras, como por ejemplo, en la expresión entre los arbustos, en donde no hay yuxtaposición mental de dos elementos para conformar el landmark de la preposición.

Nótese también la naturaleza conceptual de los elementos elaboradores tales como \{COSA\} o \{SUBSTANCIA\}. Las estructuras gramaticales parecen ser ampliamente compartidas, no así la naturaleza conceptual de los elementos elaboradores del landmark.

\begin{tabular}{|c|l|l|l|}
\cline { 2 - 4 } \multicolumn{1}{c|}{} & \multicolumn{1}{c|}{ Coordinación tipo-y } & \multicolumn{1}{c|}{ Coordinación tipo-o } & Sin estructura de coordinación \\
\hline Between & Between you and me & $\begin{array}{l}\text { You have to choose between } \\
\text { going by car or train }\end{array}$ & $\begin{array}{l}\text { There's similarity between } \\
\text { these products }\end{array}$ \\
\hline Among & $\begin{array}{l}\text { The company ranks } \\
\text { second among spirits } \\
\text { producers and marketers. }\end{array}$ & $\begin{array}{l}\text { There were no reports of } \\
\text { deaths among the dugong or } \\
\text { turtles }\end{array}$ & $\begin{array}{l}\text { The cell phone was hidden } \\
\text { among the weed }\end{array}$ \\
\hline Amid & Amid happiness and sadness & $\begin{array}{l}\text { no lo manifiesta } \\
\text { aparentemente }\end{array}$ & Amid the financial gloom \\
\hline
\end{tabular}

\subsection{ORGANIZACIÓN ESPACIAL DE LA PREPOSICIÓN ENTRE}

La preposición entre, siguiendo a Trujillo (1971), es caracterizada por presentar límite de extensión, fronteras, y ausencia de orientación, lo cual es también aplicable a usos y entendimiento espacial de sus equivalentes ingleses. Lo que tiene la preposición entre en particular es su capacidad de cubrir prácticamente todo el campo semántico de sus tres equivalentes ingleses. Esta particularidad nos indica que las proto-escenas (al igual que los parámetros derivados) de between, among y amid son las mismas que estructuran, esta vez conjuntamente, la base conceptual de entre. Esta estructuración hace que el landmark preposicional de entre pueda establecer correspondencias con el perfil de un nominal de tipo contable, masa plural y masa no plural. En otras palabras, el landmark de entre no discrimina frente a sus elementos elaboradores: tiene tendencias semánticas ${ }^{26}$ con cualquier tipo de sustantivo.

${ }^{26}$ Para ver detalles en tendencias semánticas (y formales), véase Evans (2009). 
Para evidenciar dicho rango semántico de entre, consideremos tres ejemplos extraídos de la base de datos Spanish Web 2011 (esTenTen11, Eu + Am):

(12) Anthony Reynolds, vocalista de Jack, se hizo esperar hasta subir al escenario, apareciendo de entre el público botella de vino en mano y ataviado con sus gafas de sol.

[TRAYECTORIA INTERIOR/EXTERIOR]

(13) Despertarme sin horarios, pero con tiempo a disfrutar de la mañana, disfrutar el aire que pasa a través de la ventana antes de levantarme, revolviéndome entre las sabanas... preparar el desayuno a mis acompañantes; zumo de naranja natural, un poco de fruta y unos huevos con beicon muy hecho...mmm... [OCLUSIón]

(14) Esta especie de estrecho ha recibido el nombre de Paso del Drake y, además de ser prueba fehaciente de la separación lenta pero continua entre las placas de la Antártida y de Sudamérica, es responsable de que una gran cantidad de agua muy fría circule alrededor del continente antártico, provocando su aislamiento térmico y repercutiendo en el clima global

[SEPARACIÓN/LOCACIÓN]

Los ejemplos dados arriba han sido puestos con un mayor contexto lingüístico de manera que se puede apreciar en detalle la semántica situada que entre adquiere en cada uso y cómo su base conceptual, mostrada en la figura 7 de abajo, puede ayudarnos a comprender sus zonas activas las cuales son contexto-dependientes.

En el ejemplo (12), entre adquiere un comportamiento atemporal complejo debido a que, juntamente con la preposición de, contribuye con un escaneo secuencial en la cláusula apareciendo de entre el público. El sujeto en (12) estaba en una multitud de gente, lo que implica la activación del parámetro de rodear (véase figura 7 debajo). Estar en una multitud implica 'estar incluido como constituyente de un cúmulo de personas', por lo que también hay activación del parámetro de inclusión. A esto se le podría sumar la activación de oclusión, debido a la consecuencia fenomenológica de obstaculización visual frente a una cosa o persona (figura), la cual está ubicada "entre muchas cosas o personas" (fondo). Finalmente, los parámetros de homogeneidad y proximidad también reciben activación debido al carácter homogéneo frente a lo que es "una masa de gente" y la proximidad interpersonal que implica salir de esta, como el sujeto en (12). 
El ejemplo (13) destaca otra faceta de entre, la cual tiene que ver mayoritariamente con oclusión. El compósito estructural revolviéndome entre las sabanas sirve de complemento informativo del espacio discursivo actual que trata acerca de una rutina matutina ideal de una persona. Oclusión en (13) va relacionado al hecho de que una persona que está acostada y tapada con sabanas es parcialmente (o completamente) ocluida por dicho acto. Hay que considerar también nociones metafóricas que evoca la frase preposicional entre las sabanas. El sujeto en (13) no está literalmente entre las sabanas, a menos que esté enrollado en ellas. A esto le sigue que no habría que descartar una activación y extensión de rodear (y posiblemente inclusión) en la semántica situada de entre en (13).

El ejemplo (14) hace que la preposición entre destaque el parámetro de separación, en el caso de entre las placas de hielo de la Antártida y Sudamérica. El perfil del nominal las placas establece links de correspondencias con el trajector de entre y lo elabora, mientras que los perfiles de Antártida y Sudamérica elaboran conjuntamente el landmark preposicional. Nótese que los elementos del landmark también proveen de una semántica de [LOCACIÓN], ya que sabemos que se trata del hemisferio sur del planeta.

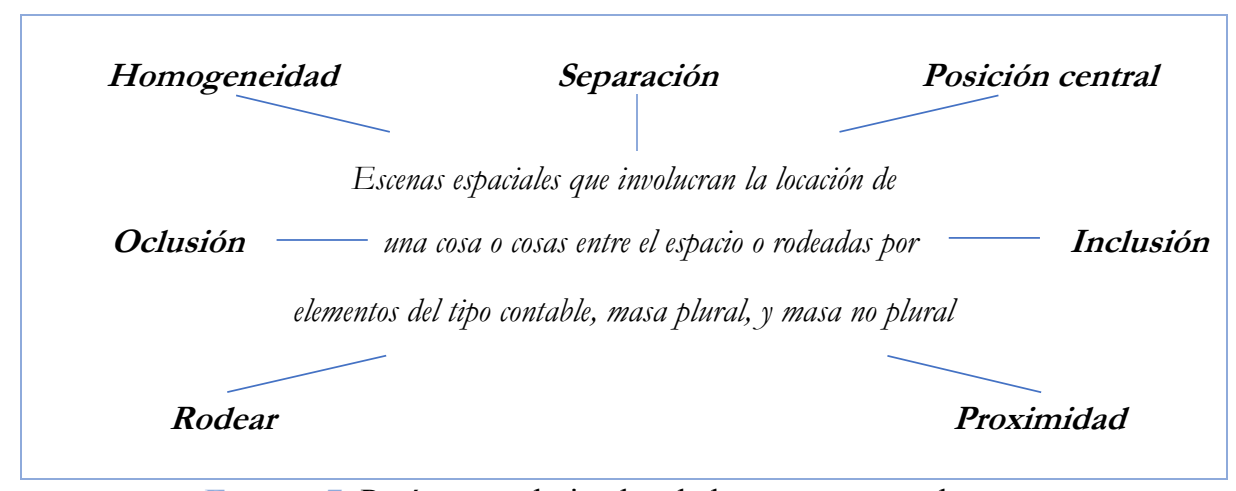

Figura 7. Parámetros derivados de la proto-escena de entre

\subsubsection{Extensión semántica y comportamiento temporal de entre}

Ahora procederemos a observar cómo la base conceptual propuesta para entre nos ayuda a entender concepciones no espaciales y temporales. Para ello, consideremos 
los siguientes ejemplos extraídos del corpus Spanish Web 2011 (esTenTen11, Eu+ $A m)$ :

(15) Puedes elegir entre muchas alternativas en comida a domicilio: cajas Chicken Box Snack Delivery para compartir de a dos; caja Chicken Box Familiar para más de tres, $o$ también un Banquetazo Mega para toda la familia.

[DECISIÓN]

(16) Al final, cocinar en El Bulli se convierte en algo que se mueve entre la ciencia y el arte. «Tenemos un equipo de investigadores que trabajan buscando cosas nuevas»

[TÉRMINO MEDIO]

(17) Es cierto que el arrendador también se beneficia de reducciones, especialmente si el arrendatario tiene entre 18 y 35 años

[DEMARCACIÓN TEMPORAL]

Los ejemplos mostrados arriba, muestran el modo en que entre puede cubrir facetas semánticas sancionadas por sus equivalentes ingleses. El uso de entre en (15), el cual es equivalente al uso de between, y en algunas otras instancias al de among, se debe principalmente a la estructura esquemática de separación, la cual es proyectada al dominio no espacial de manera de poder simular cada opción como la instancia de un tipo. Estas son especificadas por la estructura de coordinación del tipo $-o$.

En (16), entre cubre la función semántica de amid en entre la ciencia y el arte (amid the science and art). Como en el caso de amid, el parámetro semántico que dirige una posible conceptualización metafórica es el de posición central, en donde el trajector (la experiencia de cocinar en el Bulli) contiene rasgos de dos espacios mentales a causa de mezcla conceptual.

En el ejemplo (17) entre funciona temporalmente en entre 18 y 35 años (between 18 and 35). El landmark preposicional, en este caso, demarca temporalmente el rango de edad para acceder a supuestas reducciones de pago. Como es el caso de between en (5), los atributos de la estructura esquemática de separación son integrados a la estructura temporal de manera de complementar y posiblemente facilitar procesos de simulación mental relacionados al dominio temporal.

\section{COMENTARIOS Y CONCLUSIONES}


El análisis presentado nos provee un acercamiento a un mejor entendimiento acerca del espectro semántico cubierto por la preposición entre y sus equivalentes ingleses. Las bases conceptuales deben siempre ser vistas como algo enciclopédico en vez de “diccionarial” (siguiendo a Langacker 1987), en donde su semántica es distribuida en contexto (Shina \& Kuteva 1995). El análisis también ofrece una metodología analítica y aplicabilidad en las áreas de enseñanza y psicolingüística. Las bases conceptuales propuestas para las cuatro preposiciones analizadas en esta investigación pueden ser aplicadas en el aula en la manera de presentar a los estudiantes de español e inglés una visión psicológicamente real y basada en fenomenología acerca del comportamiento y origen funcional espacial de las preposiciones y cómo esta configuración nos ayuda a comprender la motivación detrás del dominio no espacial, así como también, a entender la complementación conceptual hacia el dominio temporal. Estudiantes universitarios de áreas afines como filología y lingüística también podrían verse beneficiados del análisis presentado.

Las zonas activas son también comprendidas de mejor manera. Los estudiantes pueden observar cómo las zonas activas guían la semántica situada de las preposiciones. Las bases conceptuales, por otro lado, no solo permiten comprensión de imput, sino también producción (output). Los estudiantes de español e inglés pueden usar las bases conceptuales propuestas para mejorar su productividad y usos preposicionales en nuevos contextos. Esto, por supuesto, es algo que necesita validación empírica, aunque ya ha sido demostrado que el entendimiento de estructura espacio-conceptual es esencial para el aprendizaje de usos preposicionales no espaciales (Boers \& Demecheleer 1998).

Los profesores de enseñanza de inglés y español como lengua extranjera se ven favorecidos puesto que cuentan con un entendimiento más basado en percepción, acción situada e introspección (en línea con Barsalou 1999, 2008) del lenguaje espacial y su motivación en usos no espaciales y temporales. La metodología utilizada podría ser aplicada a todo el set preposicional del español o cualquier otro idioma como el inglés. Algunos autores, como Holme (2009) (véase también Littlemore 2009, Pütz 2007 y Tyler 2012), proponen un currículo de enseñanza del inglés basado en 
lingüística cognitiva. La presente investigación podría contribuir con ello al menos con las preposiciones analizadas aquí.

El análisis nos permite establecer diferencias conceptuales entre cómo un campo semántico es cubierto por una preposición española, por un lado, y tres preposiciones inglesas, por el otro. Según Pütz (2007) la atención que los profesores deben hacer al lenguaje materno es tan importante como la que se hace del lenguaje enseñado en el aula. Saber cómo categorías funcionales están distribuidas en cada lenguaje es una de las posibles cosas que los profesores de L2 deberían indicar a sus estudiantes antes de empezar a enseñar el contenido, puesto que permite una mejor recategorización de parámetros al momento de aprender el segundo idioma.

Las bases conceptuales propuestas en esta investigación presentan un desafío a la visión estática de gramática. En línea con O’ Dowd (1998), los libros tradicionales de gramática, a excepción de algunos como Cognitive English Grammar (2007), no proveen al estudiante con definiciones satisfactorias. Particularmente en el caso de las preposiciones, estas deben ser aprendidas prácticamente de memoria. Contrario a esta filosofía de gramática como algo estricto y rígido, esta línea de trabajo asume la gramática como conceptualización (Langacker 2000) de patrones cognitivos flexibles y contexto dependientes. El lenguaje es estructurado, pero también presenta un alto grado de flexibilidad.

Por último, cabe proponer recomendaciones para investigaciones futuras. Primero en la aplicabilidad pedagógica (y de formación universitaria) de las bases conceptuales propuestas para testear su eficacia con relación a la adquisición de una nueva lengua. El segundo punto, en donde la presente investigación puede ser indagada más en detalle, concierne su posible validación experimental en el área de la psicolingüística con el fin de comprobar la existencia psicológica de los parámetros semánticos que podrían habitar las bases conceptuales propuestas.

\section{BIBLIOGRAFÍA}

Allwood, J. (2003). Meaning potentials and context: Some consequences for the analysis of variation in meaning. en H. Cuyckens, R. Dirven, and J. Taylor (Eds.), Cognitive Approaches to Lexical Semantics, Berlín: Mouton de Gruyter, pp. 29-66. 


\section{SECCIÓN: LEXICOLOGÍA}

BASE CONCEPTUAL DE LA PREPOSICIÓN ENTRE Y SUS EQUIVALENTES DE LA LENGUA INGLESA BETWEEN, AMONG, Y AMID: UNA PERSPECTIVA EN LINGÜÍSTICA COGNITIVA Javier Morras Cortés

Barcelona, A. (2000). On the plausibility of claiming a metonymic motivation for conceptual metaphor. En A. Barcelona (Ed.), Metaphor and metonymy at the crossroads: A cognitive perspective, Berlín-Nueva York: Mouton de Gruyter, pp. 31-58.

Barcelona, A. (2008). Metonymy is not just a lexical phenomenon: On the operation of metonymy in grammar and discourse. En C. Alm-Arvius, N.-L. Johansson y D. C. Munich (Eds.), Selected papers from the Stockholm 2008 Metaphor Festival, Stockholm: Stockholm University Press, pp. 3-41.

Barcelona, A. (2011). Reviewing the properties and prototype structure of metonymy. En R. Benczes, A. Barcelona y F. J. Ruiz de Mendoza Ibáñez (Eds.), Defining metonymy in cognitive linguistics: Towards a consensus view, Amsterdam-Filadelfia: John Benjamins, pp. 7-57.

Barcelona, A. (en prensa). "Metonymy”. En E. Dabrowska y D. Divjak (Eds.), Handbook of Cognitive Linguistics, Berlín-Nueva York: Mouton de Gruyter.

Barsalou, L. W. (1999). Perceptual symbol systems, en Behavioral and Brain Sciences, 22, pp. 577-660.

Barsalou, L. W. (2008). Grounded cognition, en Annual Review of Psychology, 59, pp. 617-645.

Bergen, B. K. (2012). Louder than words: The new science of how the mind makes meaning, Nueva York: Basic Books.

Boers, F. y Demecheleer, M. (1998). A cognitive semantics approach to teaching prepositions, en ELT Journal, 52, pp. 197-203.

Cambridge University Press (2008). Cambridge online dictionary. Disponible [en línea]: https://dictionary.cambridge.org.

Evans, V. (2004) The Structure of Time: Language, Meaning, and Temporal Cognition, Amsterdam: John Benjamins.

Evans, V. (2009). How Words Mean: Lexical Concepts, Cognitive Models, and Meaning Construction, Oxford: Oxford University Press.

Evans, V. (2010). From the spatial to the non - spatial: The "state" lexical concepts of in, on and at. En V. Evans y P. Chilton (Eds.), Language, Cognition \& Space: The state of the art and new directions, Londres: Equinox publishing, pp. $215-248$.

Evans, V. (2013). Language and Time: A Cognitive Linguistics Approach, Cambridge: Cambridge University Press.

Evans, V. (2015). A unified account of polysemy within LCCM Theory, en Lingua, 157, pp. 100-123.

Fauconnier, G. y Turner, M. (2002). The Way we Think: Conceptual Blending and the Mind's Hidden Complexities, New York: Basic Books.

Fillmore, C. (1982). Frame semantics. En The Linguistic Society of Korea (Ed.), Linguistics in the Morning Calm. Selected Papers from SICOL-1981, Seoul: Hanshin Publishing Company, pp. 111-37.

Gallese, V. y Lakoff, G. (2005). The brain's concepts: The role of the sensory-motor system in conceptual knowledge, en Cognitive neuropsychology, 22(3/4), pp. 455-479. 


\section{SECCIÓN: LEXICOLOGÍA}

BASE CONCEPTUAL DE LA PREPOSICIÓN ENTRE Y SUS EQUIVALENTES DE LA LENGUA INGLESA BETWEEN, AMONG, Y AMID: UNA PERSPECTIVA EN LINGÜÍSTICA COGNITIVA Javier Morras Cortés

Galton, A. (2011). Time flies but space doesn't: limits to the spatialization of time, en Journal of Pragmatics, 43: pp. 695-703.

Grady, J. (1997). Foundations of Meaning: Primary Metaphors and Primary Scenes. Unpublished doctoral thesis, linguistics dept, UC Berkeley.

Hampe, B. (2005). Image schemas in cognitive linguistics: Introduction. En Hampe, B. (Ed.), From Perception to Meaning: Image Schemas in Cognitive Linguistics, Berlín-Nueva York: Mouton de Gruyter, pp. 1-14.

Herskovits, A. (1988). Spatial expressions and the plasticity of meaning. En B. Rudzka-Ostyn (Ed.), Topics in Cognitive linguistics, Amsterdam: John Benjamins, pp. 271-98.

Holme, R. (2009). Cognitive Linguistics and Language Teacbing, New York: Palgrave Macmillan.

Johnson, M. (1987). The Body in the Mind: The bodily basis of meaning, imagination, and reason, Chicago: University of Chicago Press.

Johnson, M. (2005). The philosophical significance of image schemas. En Hampe, B. (Ed.), From Perception to Meaning: Image Schemas in Cognitive Linguistics, Berlín-Nueva York: Mouton de Gruyter, pp. 15-34.

Johnson, M. (2008). The Meaning of the Body: Aesthetics of human understanding. Chicago-Londres: University of Chicago Press.

Lakoff, G. (1990). The Invariance Hypothesis: is abstract reason based on image-schemas?, en Cognitive Linguistics, 1(1), pp. 39-74.

Lakoff, G., y Johnson, M. (1980). Metaphors we Live by, Chicago: University of Chicago press.

Lakoff, G. y Johnson, M. (1999). Philosophy in the Flesh (Vol. 4), Nueva York: Basic books.

Langacker, R. W. (1987). Foundations of Cognitive Grammar: Volume I Theoretical Prerequisites, Stanford: Stanford University Press.

Langacker, R. W. (1991). Foundations of Cognitive Grammar. Volume II Descriptive Applications, Stanford: Stanford University Press.

Langacker, R. W. (2000). Grammar and Conceptualization, Berlín-Nueva York: Mouton de Gruyter.

Langacker, R. W. (2008). Cognitive Grammar: A Basic Introduction, Oxford: Oxford University Press.

Langacker, R. W. (2009). Metonymic grammar. En K. U. Panther, L. L. Thornburg y A. Barcelona (Eds.), Metonymy and Metaphor in Grammar, Amsterdam-Filadelfia: John Benjamins Publishing, pp. 45-71.

Langacker, R. W. (2012a). Linguistic manifestations of the space-time (dis) analogy. En L. Filipović y K. M. Jaszczolt (Eds.), Space and time in languages and cultures: Language, culture and cognition, AmsterdamFiladelfia, pp. 191-215. 


\section{SECCIÓN: LEXICOLOGÍA}

BASE CONCEPTUAL DE LA PREPOSICIÓN ENTRE Y SUS EQUIVALENTES DE LA LENGUA INGLESA BETWEEN, AMONG, Y AMID: UNA PERSPECTIVA EN LINGÜÍSTICA COGNITIVA Javier Morras Cortés

Langacker, R. W. (2012b). Interactive cognition: Toward a unified account of structure, processing, and discourse, en International Journal of Cognitive Linguistics, 3(2), pp. 95.

Littlemore, J. (2009). Appying Cognitive Linguistics to Second Language Learning and Teaching, Nueva York: Palgrave Macmillan.

Mandler, J. (2004). The Foundations of Mind: Origins of Conceptual Thought. Oxford: OUP.

O’ Dowd, E. (1998). Prepositions and Particles in English: A Discourse-Functional Account, Nueva York-Oxford: Oxford University Press.

Panther, K. U. (2006). Metonymy as a usage event. En G. Kristiansen, M. Achard, R. Dirven, F. J. Ruiz de Mendoza Ibáñez (Eds.), Cognitive linguistics: Current applications and future perspectives, Berlín: Mouton de Gruyter, pp. 147-185.

Panther, K. U. y Thornburg, L. L. (2009). Introduction: On figuration in grammar. En K. U. Panther, L. L. Thornburg y A. Barcelona (Eds.), Metonymy and Metaphor in Grammar, Amsterdam-Filadelfia: John Benjamins Publishing, pp. 1-44.

Peña Cerval, S. (2012). Los esquemas de imagen. En I. Ibarretxe-Antuñano y J. Valenzuela (Dirs.), Lingüística Cognitiva, Barcelona: Anthropos, pp. 69-98.

Pütz, M. (2007). Cognitive linguistics and applied linguistics. En D. Geeraerts y H. Cuyckens (Eds.), The Oxford handbook of cognitive linguistics. Oxford: Oxford University Press, pp. 1139-1159.

Radden, G. y Dirven, R. (2007). Cognitive English Grammar, Amsterdam-Philadelphia: John Benjamins Publishing Company.

Real Academia Española (2015). Diccionario de la lengua española (23 $3^{\mathrm{a}}$ ed.), Madrid: Espasa. Disponible [en línea]: http://dle.rae.es.

Schank, R. C., y Abelson, R. P. (1977). Scripts, Plans, Goals, and Understanding: An Inquiry into Human Knowledge Structures, Hillsdale, N J: Lawrence Erlbaum.

Sinha, C. y Kuteva, T. (1995). Distributed spatial semantics, en Nordic Journal of Linguistics, 18, 167-99.

Taylor. J. (2006). Polysemy in the lexicon. En G Kristiansen, M. Achard, R. Dirven y F. J. Ruiz de Mendoza Ibáñez (Eds.), Cognitive Linguistics: Current Applications and Future Perspectives. Berlin-New York: Mouton de Gruyter, pp. 145-148.

Thompson, E. (2010). Mind in Life: Biology, Phenomenology, and the Sciences of Mind, Cambridge, Massachusetts: Harvard University Press.

Trujillo, R. (1971). Notas para un estudio de las preposiciones españolas, en Thesaurus, 26, pp. 234-279.

Tyler, A. y Evans, V. (2003). The Semantics of English Prepositions: Spatial Scenes, Embodied Meaning and Cognition. Cambridge: Cambridge University Press. 
SECCIÓN: LEXICOLOGÍA

BASE CONCEPTUAL DE LA PREPOSICIÓN ENTRE Y SUS EQUIVALENTES DE LA LENGUA INGLESA BETWEEN, AMONG, Y AMID: UNA PERSPECTIVA EN LINGÜÍSTICA COGNITIVA Javier Morras Cortés

Tyler, A. (2012). Cognitive Linguistics and Second Language Learning, Nueva York y Londres: Routledge.

Vandeloise, C. (1994). Methodology and analyses of the preposition in, en Cognitive Linguistics, 5 (2): pp. 15784. 